nature. But when an explosion subsequently occurred in the mine, and traversed the whole length and breadth of the workings, which were known to be practically free from fire-damp at the time, producing the most disastrous effects, the fallacy of the conclusions drawn from the experimental results was rendered abundantly evident.

In the whole of my papers on this subject, and most pointedly in my last article on coal-dust expiosions published in Iron, in the year 1878 , I have carefully indicated that a difference was to be expected in the behaviour of dust ignited under the two conditions named. It has therefore been with feelings of considerable surprise that I have observed members of the French, English, and German Mining Commissions, and others who have investigated this subject since the publication of my first paper, one after the other pronounce some very decided opinions as to the relatively subordinate part which coaldust plays in a colliery explosion, while at the same time they were neglecting to take into account this very simple and yet all-important element.

W. GaLlOWAY

VESUVIAN ERUPTION OF FEBRUARY 4, I886

THE rent that was formed on May 2, 1885, in the upper part of the great cone (NATURE, vol. xxxii. p. 55) gave issue to lava until December 25. A small quantity again issued between January 2 and January 5, I886, after which no more made its appearance till this new outburst. In consequence of the rise of level of the magma in the chimney, the cone of eruption has grown very much during the last month.

On February 4, at about 8 p.m., lava broke forth at the foot of the old crater ring of $188 \mathrm{I}-2$ at a point bearing from the main vent about $10^{\circ} \mathrm{W}$. of $\mathrm{N}$., traversed the crater plain, which is here very narrow, in a somewhat oblique direction, and ran down the slope of the cone between north and north-north-east. 'The lava soon reached the foot of the cone, but even up till midday today, when I left the mountain, it had not yet commenced to cross the Atrio del Cavallo. The eruption took place from probably the same dyke that gave rise to that of January 9, 1884.

To-day, February 6 , the lava bubbles up like a spring at the foot of a hill, and flows for some distance in a kind of trough which it has raised on each side of itself above the level of the crater plains. After a short distance it enters one of its own tunnels to reappear again at some distance. It was very interesting to watch its welling, and from time to time the bursting of a steam bubble as big as a bucket, which would throw up splashes against the imperfect arch at the immediate exit. These splashes partly adhered to the roof and partly fell, drawing out the suspended portion into irregular strings, illustrating the formation of the stalactitic lava that is so common in lava fumaroles.

The chloride crusts in the neighbourhood were uncommonly rich in copper, so that my boot-nails were thickly plated with that metal.

The lava makes its appearance at about 100 yards from its entrance in the tunnel near the main spring, although it is now divided into two streams. The eastern, which is the largest, is I metre broad; I plunged a stick in to the depth of I metre, but the shortness of the stick and the great heat prevented me from touching the bottom. The current ran at the rate of I metre per 6 seconds, which, making allowance for viscous drag at the edges and bottom, will give an output of at least 360 cubic metres per hour, or at least 17,280 cubic metres during the 48 hours up to 8 o'clock this evening. The more western stream was 50 centimetres broad, over $I^{\circ} 20$ metres deep (as far as I could reach with my stick), and flowed at the rate of $I$ metre in 8 seconds. Giving a loss of 2 seconds of speed from drag at sides and bottom, we have an output, for 48 hours, of 10,368 cubic metres.
The two streams together would, therefore, have afforded, since the commencement of the eruption, 27,648 cubic metres.

As the altitude of the lateral ot:tlet is much more than that of May 2, both on the night of the eruption and the following one, the volcano showed the first stage of activity as judged by the appearance of the main vent.

This winter the mountain has been covered by snow several times, and to-day it extends down nearly to the level of the Observatory. During our ascent we had to walk through a stratum of about 8 inches, though much thicker in the drifts. Two-thirds of the crater and part of the cone of eruption were also covered.

I should have sent news yesterday, but, on attempting an ascent, I was driven back by wind, rain, and mist.
Naples, February 6
H. J. JOHNSTON-LAvis

\section{TIDAL FRICTION AND THE EVOLUTION OF $A$ SATELLITE}

A PAPER by Mr. James Nolan has recently appeared which is devoted to an adverse criticism of my views concerning the effects of tidal friction as a factor in the evolution of the moon and earth.

The title of the pamphlet, "Darwin's Theory of the Genesis of the Moon," I shows, I think, that the author has misconceived the scope of my work. It was not supposed that the investigation threw light on the actual mode of genesis of the moon, but was rather applicable to the subsequent history of the moon and earth. Mr. Nolan attributes to me views as to the condition of the moon immediately after her birth which do not appear a just interpretation of my writings, and although it might have been well to use more guarded expressions in some passages, the justice of his condemnation of the whole theory cannot be admitted. He sums up his case by the three following propositions:-

"(I) That the moon could not have existed bodily so near the earth as the greatest initial distance fixed.

"(2) That in any form possible there she could not have receded by the agency assigned-tidal friction.

"(3) That, if a modification be made by allowing her to have separated at a greater radius than that corresponding to a period between 2 and 4 hours, the moon would be no longer traceable to the earth's present surface on which condition the theory has been founded."

The first of these propositions is interesting, and I have to thank him for drawing my attention to it.

When a small satellite revolves about a planet with a certain proximity, the sum of the centrifugal and tidal forces may be such as to overbalance the gravitation towards the centre of the satellite. When this is the case, the satellite cannot exist as a single mass. The complete solution of the problem, concerning which $\mathrm{Mr}$. Nolan adduces certain elementary considerations, is of extreme difficulty. At present I do not wish to go into this question, but shall consider the point on another occasion. It may, however, be admitted that the moon could not subsist as a single continuous body with its surface in contact with the earth.

On p. 4 he quotes a passage from the abstract of one of the papers (Proc. R.S., No. 200, 1879), which must be surrendered ; it is as follows:-

"The coincidence is noted in the paper that the shortest period of revolution of a fluid mass of the same mean density as the earth, which is consistent with an eliipsoidal form of equilibrium is 2 hours and 24 minutes ; and if the moon were to revolve about the earth with this periodic time, the surfaces of the two bodies would be almost in contact with one another."

Now, since 1879 it has been shown by Sir William Thomson that the ellipsoidal form referred to could not

I Geo. Robertson and Co., Melbourne, Sydney, Adelaide, and Brisbane, 1885. Pp. 16. 